BULLETIN OF THE

AMERICAN MATHEMATICAL SOCIETY

Volume 79, Number 2, March 1973

\title{
LOCAL SPECTRAL MAPPING THEOREMS
}

\section{BY ROBERT G. BARTLE AND CONSTANTINE A. KARIOTIS ${ }^{1}$ \\ Communicated September 26, 1972}

This note is concerned with various "localized versions" of the spectral mapping theorem (cf. [3, VII.3.11]) for bounded linear operators in a complex Banach space. The details of the proof and some additional results will be published elsewhere.

Let $X$ be a complex Banach space and let $T \in B(X)$, the Banach algebra of all bounded linear operators on $X$. We recall (cf. [2, p. 1], [3, p. 1931]) that if $T$ has the single-valued extension property then there exist a maximal open set $\rho_{T}(x)$ containing $\rho(T)$ and a unique holomorphic function $\tilde{x}_{T}: \rho_{T}(x) \rightarrow X$ such that $(\lambda I-T) \tilde{x}_{T}(\lambda)=x$ for all $\lambda \in \rho_{T}(x)$. The complementary set $\sigma_{T}(x)=C-\rho_{T}(x)$, which we call the local spectrum of $x$ (with respect to $T$ ), is compact and is contained in $\sigma(T)$, the spectrum of $T$. If $F \subseteq C$ is closed we introduce the spectral manifold $X_{T}(F)=\left\{x \in X: \sigma_{T}(x) \subseteq F\right\}$.

THEOREM 1. Let $f$ be holomorphic on a neighborhood of $\sigma(T)$ and suppose that $T$ and $f(T)$ have the single-valued extension property. Then $f\left(\sigma_{T}(x)\right)=$ $\sigma_{f(T)}(x)$ for all $x \in X$.

This result ${ }^{2}$ was proved by the second-named author in his dissertation [4]. The proof given there is similar to the proof of a theorem due to Colojoară and Foias ([1], [2, p. 71]). In fact, the conclusion of Theorem 1 is equivalent to the condition that if $F$ is a closed subset of $\sigma(f(T))=f(\sigma(T))$, then

$$
X_{f(T)}(F)=X_{T}\left(f^{-1}(F)\right) .
$$

If $T \in B(X)$ and $Y$ is a (closed) subspace of $X$ which is invariant under $T$, then the spectrum of the restriction $T \mid Y$ may be either smaller or larger than $\sigma(T)$. It is often desirable to limit attention to subspaces which do not increase the spectrum under restriction (e.g., ultra-invariant subspaces).

THEOREM 2. Let $Y$ be a subspace invariant under T such that $\sigma(T \mid Y) \subseteq \sigma(T)$ and let $f$ be holomorphic on a neighborhood of $\sigma(T)$. Then $Y$ is invariant

AMS(MOS) subject classifications (1970). Primary 47A10, 47A60; Secondary 47A15.

${ }^{1}$ The authors were partially supported by a grant from the National Science Foundation.

2 ADDED IN PROOF. After this note was communicated we discovered this theorem with a similar proof in C. Apostol, Teorie spectrală şi calcul funcţional, Stud. Cerc. Mat. 20, no. 5 (1968), 635-668. 
under $f(T), f(T) \mid Y=f(T \mid Y)$, and

$$
f(\sigma(T \mid Y))=\sigma(f(T \mid Y))=\sigma(f(T) \mid Y) \subseteq f(\sigma(T)) .
$$

If $Y$ is an invariant subspace for $T$ such that $\sigma(T \mid Y) \subseteq \sigma(T)$ and if $y \in Y$, then $\sigma_{T}(y) \subseteq \sigma_{T \mid Y}(y)$, but this inclusion can be proper. The next result holds for subspaces for which equality holds (e.g., spectral maximal spaces).

THEOREM 3. Suppose that $Y$ is a subspace invariant under $T$ such that $\sigma_{T \mid Y}(y)=\sigma_{T}(y)$ for all $y \in Y$. Let $f$ be holomorphic on a neighborhood of $\sigma(T)$ and let $T$ and $f(T)$ have the single-valued extension property. If $y \in Y$, we have

$$
\sigma_{f(T) \mid Y}(y)=\sigma_{f(T \mid Y)}(y)=f\left(\sigma_{T \mid Y}(y)\right)=f\left(\sigma_{T}(y)\right)=\sigma_{f(T)}(y) .
$$

If $T \in B(X)$ and $Y$ is a (closed) subspace of $X$ which is invariant under $T$ and if $T / Y$ is the operator in the quotient space $X / Y$ defined by $(T / Y)[x]=$ [TX], then the spectrum of $T / Y$ may be either smaller or larger than $\sigma(T)$. It is often desirable to limit attention to subspaces which do not increase the spectrum under quotients (e.g., ultra-invariant subspaces).

THEOREM 4. Let $Y$ be a subspace invariant under $T$ such that $\sigma(T / Y) \subseteq$ $\sigma(T)$ and let $f$ be holomorphic on a neighborhood of $\sigma(T)$. Then $Y$ is invariant under $f(T), f(T) / Y=f(T / Y)$, and

$$
f(\sigma(T / Y))=\sigma(f(T / Y))=\sigma(f(T) / Y) \subseteq f(\sigma(T)) .
$$

In addition, if $T, f(T), T / Y$, and $f(T) / Y$ have the single-valued extension property and $x \in X$, then we have

$$
\sigma_{f(T) / Y}([x])=\sigma_{f(T / Y)}([x])=f\left(\sigma_{T / Y}([x])\right) \subseteq f\left(\sigma_{T}(x)\right)=\sigma_{f(T)}(x) .
$$

\section{REFERENCES}

1. I. Colojoară and C. Foiaş, The Riesz-Dunford functional calculus with decomposable operators, Rev. Roumaine Math. Pures Appl. 12 (1967), 627-641. MR 39 \# 3334.

2. - Theory of generalized spectral operators, Gordon and Breach, New York, 1968

3a. N. Dunford and J. T. Schwartz, Linear operators. I: General theory, Pure and Appl. Math., vol. 7, Interscience, New York, 1958. MR 22 \# 8302.

3b. _ Linear operators. III : Spectral operators, Interscience, New York, 1971.

4. C. A. Kariotis, Spectral properties of certain classes of operators, Dissertation, University of Illinois, Urbana-Champaign, Ill., 1966.

Department of Mathematics, University of Illinois, Urbana-Champaign, Illinois 61801.

Department of Mathematics, Georgia Southern College, Statesboro, Grorgia 30458. 